\title{
A Hybrid Cognitive Transceiver Architecture: Sensing-Throughput Tradeoff
}

\author{
Shree Krishna Sharma, Symeon Chatzinotas, and Björn Ottersten \\ SnT - securityandtrust.lu, University of Luxembourg \\ Email: $\{$ shree.sharma, symeon.chatzinotas, bjorn.ottersten\}@uni.lu
}

\begin{abstract}
Spectrum Sensing (SS) and underlay have been considered as two important techniques for enabling the spectral coexistence of the licensed and unlicensed wireless communication systems. The SS only approach ignores the interference tolerance capability of the Primary Users (PUs) while assuming the bursty PU traffic whereas the possibility of having secondary transmission with full power is neglected in an underlay based approach. To address this, it's crucial to investigate suitable hybrid spectrum awareness and transmission strategies which can overcome the aforementioned drawbacks and achieve higher secondary throughput while protecting the PUs. In this context, we propose a hybrid cognitive transceiver which combines the SS approach with the power control based underlay approach. Furthermore, we evaluate the performance of the proposed hybrid approach considering both periodic sensing and simultaneous sensing/transmission schemes. Moreover, sensing-throughput tradeoff for the proposed hybrid approach is investigated and the performance is compared with the conventional SS only approaches in terms of the achievable throughput. It is shown that the proposed scheme can achieve a significant improvement in the secondary throughput over the conventional approaches while respecting the interference constraints of the PUs.
\end{abstract}

\section{INTRODUCTION}

The demand for broadband wireless spectrum is increasing due to a rapidly expanding market of wireless broadband and multimedia users and applications. However, the available usable spectrum has become scarce due to spectrum segmentation and current static frequency allocation policies. In this context, Cognitive Radio (CR) communications is considered a promising solution in order to address the spectrum scarcity problem since it allows the spectral coexistence of two wireless systems using different spectrum awareness and advanced interference mitigation techniques. The most common spectrum sharing techniques in the literature can be categorized into interweave or Spectrum Sensing (SS), underlay, overlay and database techniques $[1,2]$. In SS only techniques, Secondary Users (SUs) are allowed to transmit in a particular licensed band whenever Primary Users (PUs) do not use that band, whereas in underlay techniques, SUs are allowed to transmit even if the PU channel is active as long as they meet the interference constraint of the PUs. In the context of SS, there exist several techniques such as Energy Detection (ED), matched filter based detection, cyclostationary feature based detection, correlation based detection, and eigenvalue based detection [3,4]. Under the underlay CR literature, there exist several interference mitigation methods such as interference alignment, beamforming, power control, and exclusion zone based approaches [5-7].

Most of the existing spectrum sharing approaches focus either on SS or underlay-based approach. In the context of SS, better sensing accuracy can be achieved with the help of a longer sensing duration. However, it reduces the aggregate throughput of the CR system since the data transmission time is reduced while increasing the sensing duration for a half duplex CR transceiver [8]. Furthermore, the throughput of the CR system can be increased by allowing higher miss detection in the CR systems i.e., by reducing the sensing time, however, it drastically degrades the Quality of Service (QoS) of the primary link. From the PU's perspective, the higher the probability of detection, the higher will be the probability that it is not interfered with whereas from the SU's perspective, the lower the probability of false alarm, the higher the spectrum utilization and hence the higher the throughput. In this context, there exists a sensing-throughput tradeoff in half duplex CR systems and this issue has received important attention in the CR literature [8-13].

The SS approach ignores the interference tolerance capability of the PUs while assuming the bursty PU traffic whereas the possibility of having secondary transmission with full power is neglected in the underlay based approach [15]. More specifically, the underlay alone approach can not detect the activity or inactivity of the PUs in a particular band and hence can not utilize the idle bands efficiently. On the other hand, the SS alone approach does not allow the SUs to transmit in a particular frequency band when the PU is active in that band. To address this, it's crucial to investigate suitable hybrid spectrum awareness and transmission strategies which can overcome the drawbacks of both the techniques and achieve higher secondary throughput while protecting the PUs. In the context of this hybrid approach, the contribution in [11] studies the transmission mode selection with channel switching in hybrid underlay/overlay systems based on the achievable throughput ratio between underlay and SS transmissions and the PU traffic statistics. In the similar context, the recent contribution in [15] proposes a hybrid strategy, which combines spectrum sensing and underlay schemes and utilizes a double energy-threshold method in order to switch between full and partial access modes dynamically.

\section{A. Contributions}

In this paper, we propose a hybrid architecture for a $\mathrm{CR}$ transceiver which combines the aforementioned advantages of 
both SS and underlay techniques. In the proposed approach, we consider a power control based underlay scheme, which selects the secondary transmit power based on the interference constraints of the PUs. It should be noted that authors in $[8,11]$ analyze the sensing-throughput tradeoff for SS only approach without considering the hybrid context considered in this paper. Furthermore, authors in [11] analyze the switching strategies for underlay and SS techniques considering the throughout ratio between underlay and SS techniques. However, in [11], the total throughput analysis of the hybrid approach is not considered and no sensing errors have been included in the analysis. However, in practice, there always exist sensing errors and we have to take them into account. In this context, we analyze the achievable throughput of the proposed hybrid CR transceiver by taking account of throughput from both SS and underlay based approaches and considering the effect of sensing errors in the analysis, which is the main contribution of this paper. Furthermore, we analyze the sensing-throughput tradeoff for the proposed hybrid spectrum awareness and transmission approach. According to authors' knowledge, the issue of sensing-throughput tradeoff for the considered hybrid approach has not been considered in the literature. Moreover, we study the proposed framework in the context of the periodic sensing frame structure [8] and simultaneous sensing/transmission (Tx) frame structure [9] considered in the literature. In the periodic SS, the CR senses the PU band for a short period of time at the beginning of each frame and then dedicates the remaining frame time for data transmission whereas in the simultaneous sensing/Tx, the CR senses and transmits data simultaneously as illustrated in [11]. The performance of the proposed approach is compared with SS only approaches with both considered frame structures.

\section{B. Structure}

The remainder of this paper is organized as follows: Section II reviews two SS approaches discussed in the literature. Section III proposes a hybrid architecture for a CR transceiver. Section IV analyzes the throughput of conventional sensing approaches using the ED scheme. Section V presents throughput analysis for the proposed scheme. Section VI evaluates and compares the performance of the considered approaches with the help of numerical results. Finally, Section VII concludes the paper.

\section{Existing SEnsing Approaches}

\section{A. Periodic Spectrum Sensing}

The frame structure of the CR with the periodic SS is shown in Fig. 1. In this approach, the CR sensing module performs SS for a short period, let us denote by $\tau$ and transmits data for the remaining $(T-\tau)$ duration, $T$ being the frame duration [8]. It is assumed that the PU activity remains constant during one frame period. In practice, either synchronization is required between primary and secondary transmissions or the SU frame must be much shorter than the PU frame for the above assumption to be true. This further implies the assumption that the PU has a periodic and known transmit duty cycle.

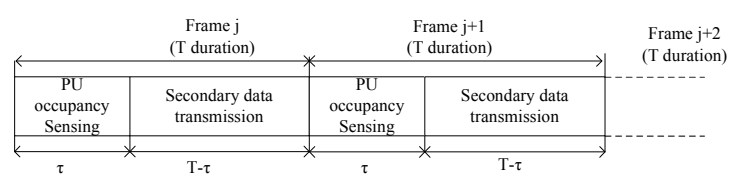

Fig. 1: Secondary frame structure for periodic spectrum sensing

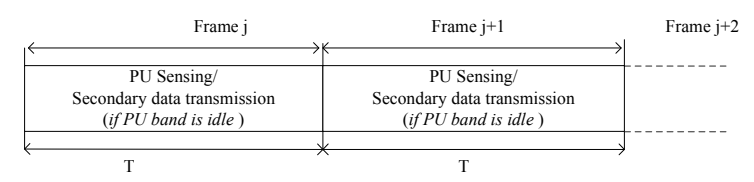

Fig. 2: Secondary frame structure for simultaneous sensing/Tx

This scheme can also be referred as a half duplex SS scheme since the SUs do not perform sensing and data transmission simultaneously. Furthermore, the SUs are not able to detect the PU's status when the SUs are transmitting, hence causing interference to the PUs. In this frame structure, there exists an inherent tradeoff between sensing time and the secondary throughput as noted in various literature $[8,12]$ and finding an optimal sensing time for the $\mathrm{CR}$ is an issue.

\section{B. Simultaneous Sensing and Transmission}

The frame structure of the CR with simultaneous sensing and transmission is shown in Fig. 2. In this case, we can consider the following two scenarios

- As in [9], it can be assumed that a CR transmits and receives interchangeably over the time. When a $C R$ is operating in the receive mode, it receives the combination of the PU signals as well as the SU signal. The received signal is decoded at the $\mathrm{CR}$ receiver to extract the secondary signal and the residue part is the PU signal plus noise. By comparing the residue part with the predefined threshold, the CR takes the decision about the presence or the absence of the PU activity and can use this decision to transmit or not in the next frame. However, in this case, we should make an assumption that the PU activity does not change during the duration of twice the frame period i.e., $2 \mathrm{~T}$.

- Another scenario is a full duplex scenario in which the $\mathrm{CR}$ transmission is non-time slotted as considered in [16]. In this case, two Radio Frequency (RF) chains are required at the $\mathrm{CR}$ receiver as depicted in Fig. 4. This scheme can accommodate the condition that the PU may change its state during a single SU frame period. Since the full duplex transmission in wireless communications has recently received important attention [16], this concept seems to be promising for full duplex $\mathrm{CR}$ nodes of future wireless communications.

In both of the above scenarios, the sensing and data transmission take place for the whole duration of the secondary frame and no sensing-throughput tradeoff exists [9]. 


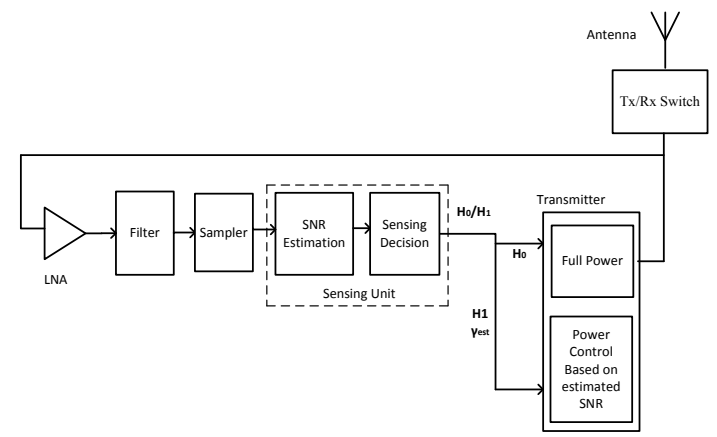

Fig. 3: Proposed half duplex architecture for a CR transceiver

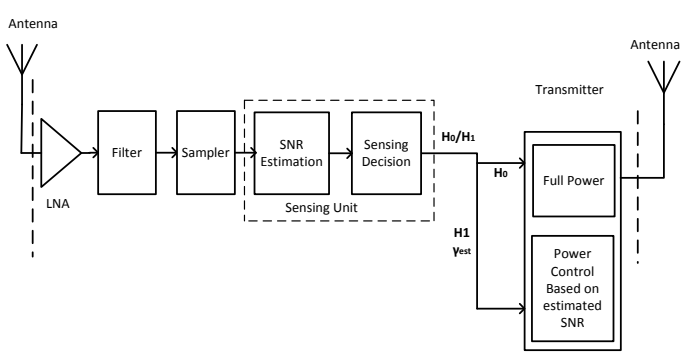

Fig. 4: Proposed full duplex architecture for a CR transceiver

\section{Proposed Hybrid Architecture For CR TRANSCEIVER}

Figure 3 presents an architecture for the CR transceiver with a single RF chain. Since this architecture consists of a single RF chain, it is capable of either sensing or transmitting data during a single time slot, hence, can be called as half duplex structure in general. However, if a pair of CR nodes i.e., a CR transmitter and a CR receiver, operates in a half duplex communication mode in such a way that they transmit and receive interchangeably over a time, this half duplex structure can function as simultaneous sensing and transmission framework mentioned in Section II-B. In the proposed architecture in Fig. 3 , the received signal is firstly passed through a Low Noise Amplifier (LNA) before further processing in the receiver. Subsequently, the amplified signal is passed through the filter, sampler and then the sensing unit. Based on the sensing requirements and the availability of knowledge about PU signal, channel and the noise variance, the sensing unit may implement any of the signal detection techniques mentioned in Section I. In this work, we focus on the ED technique due to its low implementation complexity and realize it using SNR estimation and sensing decision blocks as shown in Figs. 3 and 4. The SNR of the received PU signal can be estimated with the help of various power spectrum and SNR estimation techniques [14, 18-22]. Subsequently, based on the estimated PU SNR, let us denote by $\gamma_{\text {est }}$, the sensing unit takes a decision about the presence or absence of the PU and the decision can trigger the power control block directly in order to transmit with full power in its data transmission slot if the noise only hypothesis $\left(H_{0}\right)$ is selected. Alternatively, it can trigger the power control block along with the information about the estimated SNR if the signal plus noise hypothesis $\left(H_{1}\right)$ is selected. Based on the estimated SNR, the power control technique calculates the amount of the transmit power while considering the interference constraint of the PU as described later in Section V-A.

Figure 4 presents the proposed transceiver architecture for the full duplex scenario mentioned in Section II-B. This architecture functions in the similar way as the half duplex structure shown in Fig. 3 except the fact that it contains two separate RF chains in order to sense and transmit data simultaneously. It should be noted that in this study, we focus on the narrowband context meaning that detection of a single PU channel as in various literature $[8,9,12]$.

\section{Throughrut Analysis For CONVEntional APPROACHES}

Let us consider a frequency band $W$ with the center carrier frequency $f_{c}$ and we are interested in detecting the presence or absence of the PU signals in this band. Let us consider that the received signal at the $\mathrm{CR}$ receiver is sampled at the sampling frequency of $f_{s}$. Under the $H_{1}$ hypothesis, the discrete received signal at the $\mathrm{CR}$ receiver can be represented as

$$
y(n)=x(n)+z(n),
$$

where $x(n)$ is the transmitted signal at the $n$th time instant and $z(n)$ denotes the Additive White Gaussian Noise (AWGN) noise. Similarly, under the $H_{0}$ hypothesis, the above equation reduces to $y(n)=z(n)$. Let us consider that the transmitted signal $x(n)$ is an independent and identically distributed (i.i.d.) random process with mean zero and variance $\mathbb{E}[x(n)]^{2}=\sigma_{x}^{2}$ and the noise $z(n)$ is a Gaussian i.i.d. random process with zero mean and variance $\mathbb{E}[z(n)]^{2}=\sigma_{z}^{2}$. Furthermore, we assume that the PU signal $x(n)$ is independent of the noise $z(n)$. As mentioned earlier in Section III, we consider the ED technique for SS purpose. Let $\tau$ be the sensing time and $N$ be the number of samples collected within this duration i.e., $N=\tau f_{s}$. The test statistic for the ED technique is given by $D=\frac{1}{N} \sum_{n=1}^{N}|y(n)|^{2}$. It can be noted that the test statistic $D$ is a random variable and under the $H_{0}$ hypothesis, its Probability Density Function (PDF) follows a Chi-squared distribution with $2 N$ degrees of freedom for the complex valued case [4]. For very large values of $N$, the PDF of $D$ can be approximated by a Gaussian distribution with mean $\mu=\sigma_{z}^{2}$ and variance $\sigma_{0}^{2}=\frac{1}{N}\left[\mathbb{E}[z(n)]^{4}-\sigma_{z}^{2}\right]$ [8]. Furthermore, we assume the perfect knowledge of noise variance for the considered ED technique as in various ED related literature. In this context, the test statistic $D$ is related to the estimated SNR $\left(\gamma_{\text {est }}\right)$ depicted in Figs. (3) and (4) as follows: $D=$ $\gamma_{\text {est }} \sigma_{z}^{2}$. Using binary hypothesis testing, the expressions for probability of false alarm $\left(\mathcal{P}_{f}\right)$ and probability of detection $\left(\mathcal{P}_{d}\right)$ can be computed by [23]; $\mathcal{P}_{f}=\operatorname{Pr}\left(D>\lambda \mid H_{0}\right)$, and $\mathcal{P}_{d}=\operatorname{Pr}\left(D>\lambda \mid H_{1}\right)$, where $\lambda$ is the sensing threshold. For Circularly Symmetric Complex Gaussian (CSCG) noise case, the expression for $\mathcal{P}_{f}$ can be written as [8]

$$
\mathcal{P}_{f}(\lambda, \tau)=Q\left(\left(\frac{\lambda}{\sigma_{z}^{2}}-1\right) \sqrt{\tau f_{s}}\right),
$$


where $Q($.$) is the complementary distribution function$ of the standard Gaussian random variable i.e., $Q(x)=$ $\frac{1}{\sqrt{2 \pi}} \int_{x}^{\infty} \exp \left(\frac{-t^{2}}{2}\right) d t$. Similarly, under the $H_{1}$ hypothesis, the expression for $\mathcal{P}_{d}$ is given by

$$
\mathcal{P}_{d}(\lambda, \tau)=Q\left(\left(\lambda / \sigma_{z}^{2}-\gamma_{p}-1\right) \sqrt{\frac{\tau f_{s}}{2 \gamma_{p}+1}}\right),
$$

where $\gamma_{p}$ is the PU SNR at the secondary sensor. Let $\overline{\mathcal{P}}_{d}$ be the target probability of detection required by the detector. Combining (2) and (3), $\mathcal{P}_{f}$ is related to $\overline{\mathcal{P}}_{d}$ as follows [8]

$$
\mathcal{P}_{f}=Q\left(\sqrt{\left(2 \gamma_{p}+1\right)} Q^{-1}\left(\overline{\mathcal{P}}_{d}\right)+\sqrt{\tau f_{s}} \gamma_{p}\right)
$$

\section{A. Periodic Sensing}

Let $C_{1}$ and $C_{0}$ be the throughput of the secondary network in the presence and the absence of the PU respectively. Furthermore, let $\gamma_{s}$ and $\gamma_{p}$ be the SNRs of the received power of the SU and the PU measured at the secondary receiver respectively and are given by $\gamma_{s}=\frac{P_{s}}{\sigma^{2}}$ and $\gamma_{p}=\frac{P_{p}}{\sigma_{z}^{2}}$, where $P_{s}$ is the received power of the SU and $P_{p}$ is the interference power of the PU measured at the secondary receiver. Assuming the PU's and the SU's signals to be Gaussian, white and independent of each other, the expressions for $C_{0}$ and $C_{1}$ can be written as [8]

$$
\begin{aligned}
C_{0} & =\log _{2}\left(1+\gamma_{s}\right), \\
C_{1} & =\log _{2}\left(1+\frac{\gamma_{s}}{1+\gamma_{p}}\right) .
\end{aligned}
$$

We define $\mathcal{P}\left(H_{0}\right)$ as the probability of the PU being inactive, and $\mathcal{P}\left(H_{1}\right)$ as the probability of the PU being active in such a way that $\mathcal{P}\left(H_{0}\right)+\mathcal{P}\left(H_{1}\right)=1$. When there is perfect detection under the $H_{0}$ hypothesis, i.e., $\mathcal{P}_{f}=0$, then the throughput of the secondary link is given by $\frac{T-\tau}{T} C_{0}$. Since in practice, we do not have perfect detection and there always exists some probability of false alarm $P_{f}$, the probability of having perfect detection under the $H_{0}$ hypothesis is given by $\left(1-\mathcal{P}_{f}(\lambda, \tau)\right) \mathcal{P}\left(H_{0}\right)$. Similarly, in case of miss detection under the $H_{1}$ hypothesis, the throughput of the secondary link is given by $\frac{T-\tau}{T} C_{1}$ and the probability of having such a situation can be written as: $\left(1-\mathcal{P}_{d}(\lambda, \tau)\right) \mathcal{P}\left(H_{1}\right)$.

Taking the above definitions into account, the total throughput for the secondary network is given by

$$
R(\lambda, \tau)=R_{0}(\lambda, \tau)+R_{1}(\lambda, \tau),
$$

where the values of $R_{0}(\lambda, \tau)$ and $R_{1}(\lambda, \tau)$ can be calculated using the following expressions

$$
\begin{aligned}
& R_{0}(\lambda, \tau)=\frac{T-\tau}{T}\left(1-\mathcal{P}_{f}(\lambda, \tau)\right) \mathcal{P}\left(H_{0}\right) C_{0}, \\
& R_{1}(\lambda, \tau)=\frac{T-\tau}{T}\left(1-\mathcal{P}_{d}(\lambda, \tau)\right) \mathcal{P}\left(H_{1}\right) C_{1} .
\end{aligned}
$$

\section{B. Simultaneous Sensing/Transmission}

In this scenario, the sensing duration is $T$ instead of $\tau$ in the periodic SS approach as described in the previous subsection. For a target probability of detection $\bar{P}_{d}$, the probability of false alarm expression in (4) for the ED technique can be written as

$$
\mathcal{P}_{f}(T)=Q\left(\sqrt{\left(2 \gamma_{p}+1\right)} Q^{-1}\left(\overline{\mathcal{P}}_{d}\right)+\sqrt{T f_{s}} \gamma_{p}\right) .
$$

Therefore, the total throughput of the simultaneous sensing and transmission approach can be written as

$$
R(\lambda, T)=R_{0}(\lambda, T)+R_{1}(\lambda, T),
$$

where the values of $R_{0}(\lambda, T)$ and $R_{1}(\lambda, T)$ can be calculated using the following expressions

$$
\begin{aligned}
& R_{0}(\lambda, T)=\left(1-\mathcal{P}_{f}(\lambda, T)\right) \mathcal{P}\left(H_{0}\right) C_{0} \\
& R_{1}(\lambda, T)=\left(1-\mathcal{P}_{d}(\lambda, T)\right) \mathcal{P}\left(H_{1}\right) C_{1} .
\end{aligned}
$$

\section{Throughrut ANALYSIS FOR THE PROPOSED APPROACH}

In the proposed approach, the SU transmission strategy is based not only on the sensing decision but also on the power control based underlay mechanism. For the power control purpose, the sensing unit provides information about the estimated SNR to the power control block if the $H_{1}$ hypothesis is decided as noted in Figs. 3 and 4. Let $\lambda$ be the sensing threshold, and $P_{\text {est }}$ be the estimated power i.e., $P_{\text {est }}=\gamma_{e s t} \sigma_{z}^{2}$, which can be obtained based on suitable power spectrum or SNR estimation techniques [14,18-20]. We assume that the SUs have the knowledge of the interference threshold of the PUs, which can be known based on the technical standards and regulations. We employ the following transmission strategy for the secondary transmission. Under the $H_{0}$ hypothesis, (i) if the hypothesis is satisfied i.e., $P_{\text {est }}<\lambda$, the SUs transmit with a full power level, let us denote by $P_{\text {full }}$, (ii) if the hypothesis is not satisfied i.e., $P_{\text {est }}>\lambda$, the SUs transmit with a reduced power level, let us denote by $P_{\text {red }}$, which is calculated based on the power control mechanism described later in Section VA. In this case, even if the PU is inactive, the SU assumes the active state of the PU and transmits with $P_{\text {red }}$.

Similarly, under the $H_{1}$ hypothesis, (i) if the hypothesis is satisfied i.e., $P_{\text {est }}>\lambda$, the SUs transmit with the reduced power level $P_{\text {red }}$, (ii) if the hypothesis is not satisfied i.e., $P_{\text {est }}<\lambda$, the SUs transmit with the full power level $P_{\text {full }}$. In this case, even if the PU is active, the SU assumes the inactive state of the PU and transmits with $P_{\text {full }}$, hence causing interference to the PU. This interference is usually handled by regulations and standard as described later in Section V-A. Based on the above proposed hybrid strategy, we derive the total achievable throughput based on widely used Shannon formula. Let's introduce a new rate for the secondary link, which arises due to the partial access of the PU spectrum by the SU using the reduced power level. We denote the rate of the secondary link under the reduced power level as $C_{2}$ and define as

$$
C_{2}=\log _{2}\left(1+\frac{\gamma_{s r}}{1+\gamma_{p}}\right)
$$

where $\gamma_{s r}=\frac{P_{\mathrm{red}}}{\sigma_{z}^{2}}$. It should be noted that in (11), we assume perfect estimation of interference at the CR receiver and in the presence of interference and channel uncertainties, the performance outage constraints should be taken into consideration. In this case, our results provide upper bound on the 
achievable throughput. Subsequently, the total throughput for periodic sensing case can be calculated as

$$
R(\lambda, \tau)=R_{0}(\lambda, \tau)+R_{1}(\lambda, \tau)+R_{2}(\lambda, \tau),
$$

where the values of $R_{0}(\lambda, \tau), R_{1}(\lambda, \tau)$ and $R_{2}(\lambda, \tau)$ can be calculated using the following expressions

$$
\begin{aligned}
R_{0}(\lambda, \tau)= & \frac{T-\tau}{T}\left(1-\mathcal{P}_{f}(\lambda, \tau)\right) \mathcal{P}\left(H_{0}\right) C_{0}, \\
R_{1}(\lambda, \tau)= & \frac{T-\tau}{T}\left(1-\mathcal{P}_{d}(\lambda, \tau)\right) \mathcal{P}\left(H_{1}\right) C_{1}, \\
R_{2}(\lambda, \tau)= & \frac{T-\tau}{T} \mathcal{P}_{d}(\lambda, \tau) \mathcal{P}\left(H_{1}\right) C_{2} \\
& +\frac{T-\tau}{T} \mathcal{P}_{f}(\lambda, \tau) \mathcal{P}\left(H_{0}\right) C_{2} .
\end{aligned}
$$

It should be noted that authors in [8] and [9] have not considered the case of $R_{2}$ in their analysis. Similarly, the achievable throughput for the simultaneous sensing/Tx case can be calculated using the following expression

$$
R(\lambda, T)=R_{0}(\lambda, T)+R_{1}(\lambda, T)+R_{2}(\lambda, T),
$$

where the values of $R_{0}(\lambda, T), R_{1}(\lambda, T)$, and $R_{2}(\lambda, T)$ can be calculated as

$$
\begin{aligned}
& R_{0}(\lambda, T)=\left(1-\mathcal{P}_{f}(\lambda, T)\right) \mathcal{P}\left(H_{0}\right) C_{0} \\
& R_{1}(\lambda, T)=\left(1-\mathcal{P}_{d}(\lambda, T)\right) \mathcal{P}\left(H_{1}\right) C_{1} \\
& R_{2}(\lambda, T)=\mathcal{P}_{d}(\lambda, T) \mathcal{P}\left(H_{1}\right) C_{2}+\mathcal{P}_{f}(\lambda, T) \mathcal{P}\left(H_{0}\right) C_{2} .
\end{aligned}
$$

It should be noted from (13) and (15) that the achievable throughput for the simultaneous sensing/Tx case is higher than the achievable throughput for the periodic sensing case.

\section{A. Power control}

The aggregate secondary interference towards the PR should be below the tolerable interference threshold of the PR in order to provide sufficient primary protection. Let us denote the tolerable interference threshold of the PR by $I_{T}$. Under the proposed framework, the Secondary Transmitter (ST) may interfere with the PR in the following two cases: (i) Transmission under the $H_{1}$ hypothesis with probability $\mathcal{P}\left(H_{1}\right)\left(1-\mathcal{P}_{d}\right)$ as in [9], and (ii) Transmission under the $H_{1}$ hypothesis with probability $\mathcal{P}\left(H_{1}\right) \mathcal{P}_{d}$ under the proposed hybrid framework. More specifically, for a target $\overline{\mathcal{P}}_{d}$, there exist the following constraints for restricting interference towards the PU.

$$
\begin{array}{r}
\mathcal{P}\left(H_{1}\right)\left(1-\overline{\mathcal{P}}_{d}\right) \alpha P_{\text {red }} \leq I_{T}, \\
\mathcal{P}\left(H_{1}\right) \alpha \overline{\mathcal{P}}_{d} P_{\text {red }} \leq I_{T},
\end{array}
$$

where $\alpha$ denotes the path loss between ST and the PR. If we look carefully at the first constraint, when a SU misses the PU detection, it assumes the state as if there were no PUs and transmits with full power instead of implementing any power control. This constraint is usually handled by the regulations and standards by defining the desired probability of detection for the secondary system so that the primary system is able to tolerate the outage for the duration of the miss detection. Therefore, we focus on the second constraint in our power control mechanism as follows

$$
P_{\text {red }} \leq \frac{I_{T}}{\alpha \mathcal{P}\left(H_{1}\right) \overline{\mathcal{P}}_{d}} .
$$

The path loss $\alpha$ between ST and the PR can be estimated with the help of the estimated PU SNR and the Effective Isotropic Radiated Power (EIRP) of the primary system. The received PU SNR can be obtained by using power spectrum or SNR estimation algorithms as mentioned in Section III. Furthermore, the EIRP for the primary system can be obtained based on the power class of the primary terminals, which is known based on the specifications of wireless standards such as LTE, GSM/GPRS, EDGE etc. For this purpose, we assume that the power class of the primary terminals is known to the CR. As an example, denoting EIRP for the Primary Transmitter (PT) by $\operatorname{EIRP}_{p}$, the path loss between the ST and the PR can be calculated using the Friss transmission formula as

$$
\alpha(\mathrm{dB})=\operatorname{EIRP}_{p}(\mathrm{dBW})+G_{r}(\mathrm{~dB})-P_{\text {est }}(\mathrm{dBW}),
$$

where $G_{r}$ is the gain of the CR receive antenna. Subsequently, based on this path loss or attenuation and the interference threshold of the PU receiver, the ST adapts its transmit power based on (17). If there are multiple PUs in the system, we need to consider the worst case i.e., minimum path loss $\alpha$ in order to calculate the transmit power based on (17). For a number of PUs under a given power class, if the PU with the minimum path loss i.e., strongest estimated power is protected, all other PUs under that class can be protected. In this case, we assume that each PT only transmits during its own slot.

It should be noted that the CR estimates the received PU power, which is transmitted from the PT and hence the path loss using (18) denotes the path loss between the CR receiver and the PT. However, in practice, we need to protect the PRs from harmful interference caused by the ST. For this purpose, we can consider the following practical cases [14]. The first case assumes duplex transmission mode for the PUs i.e., each user interchangeably transmits and receives over time. If we estimate the path loss based on the strongest SNR over multiple time slots, we can also protect the weakest link assuming they have the same interference threshold. The same explanation applies for the case of multiple PU links as well. If the link with the strongest power is protected, then all the other PU links can be protected. The second scenario considers the simplex mode of transmission for the PUs and a short range wireless communication for both primary and secondary systems provided that interference levels from one system to another are at a similar level. In practice, this case arises when a spectrum resource is left completely unused within a sufficiently large network coverage area [24]. In this case, fixing secondary transmit power based on the received signal from the PT is a reasonable strategy for protecting the PR as well.

\section{NumericAl RESUlts}

In this section, we evaluate the performance of the proposed hybrid approach with the help of numerical results. We use 
TABLE I: Throughput Calculation for Various Cases

\begin{tabular}{l|c} 
Case & Reference \\
\hline Conventional Approach with Periodic Sensing & Eqn. (6) \\
Conventional Approach with Simultaneous Sensing/Tx & Eqn. (9) \\
Proposed Hybrid Approach with Periodic Sensing & Eqn. (12) \\
Proposed Hybrid Approach with Simultaneous Sensing/Tx & Eqn. (14) \\
\hline
\end{tabular}

achievable secondary throughput as a performance metric and present the variation of this metric with respect to sensing time, PU interference threshold, and the received PU SNR. We consider carrier bandwidth and the sampling frequency to be $6 \mathrm{MHz}$. Let us consider $\mathcal{P}\left(H_{1}\right)=0.2$ and the target detection probability be 0.9 as in [8]. In practice, the value of $\overline{\mathcal{P}}_{d}$ should be close to but less than 1 , especially in the low SNR region. As an example, the IEEE 802.22 cognitive Wireless Regional Area Network (WRAN) standard requires the receiver sensitivity of $-116 \mathrm{dBm}$ for sensing Digital Television (DTV) signals with $90 \%$ probability of detection [25]. Furthermore, we consider received secondary SNR as $\mathrm{SNR}_{s}=20 \mathrm{~dB}$ and we evaluate the throughput performance in low primary SNR region. Let us consider the frame duration to be $T=100 \mathrm{~ms}$. In the presented simulation results, we consider a fixed channel attenuation of $10 \mathrm{~dB}$ for the channel between the SU and the PR as in [9]. We present the corresponding throughput expressions for the considered four different cases in Table I.

Figure 5 presents throughput versus sensing time for different approaches with parameters $\left(I_{T}=-130 \mathrm{dBW}, \overline{\mathcal{P}}_{d}=0.9\right.$, $\left.T=100 \mathrm{~ms}, \mathrm{SNR}_{p}=-15 \mathrm{~dB}, \mathrm{SNR}_{s}=20 \mathrm{~dB}\right)$. From the figure, it can be noted that there exists a tradeoff between throughput and sensing time for the periodic sensing approach as noted in [8]. It can be depicted that for both conventional and the proposed hybrid approaches, the optimum sensing time is $2.5 \mathrm{~ms}$ and the proposed hybrid approach with periodic sensing achieves higher throughput in comparison to the conventional periodic sensing approach for all the considered values of the sensing duration. For example, at the sensing time of $2.5 \mathrm{~ms}$, the proposed hybrid approach with periodic sensing achieves $5.6 \mathrm{bits} / \mathrm{s} / \mathrm{Hz}$ throughput, whereas the conventional periodic sensing approach achieves only about $5.3 \mathrm{bits} / \mathrm{s} / \mathrm{Hz}$ i.e., $0.3 \mathrm{bits} / \mathrm{s} / \mathrm{Hz}$ increased throughput, which is a considerable gain. Moreover, while considering the simultaneous sensing/Tx case, the proposed hybrid approach achieves 5.76 bits/s/Hz throughput whereas the conventional approach achieves $5.45 \mathrm{bits} / \mathrm{s} / \mathrm{Hz}$ throughput. Therefore, it can be concluded that the proposed hybrid approach performs better than conventional approaches for both periodic sensing and simultaneous sensing/Tx cases.

Figure 6 presents the secondary achievable throughput versus PU interference threshold with parameters $\left(I_{T}=-130\right.$ $\mathrm{dBW}, \overline{\mathcal{P}}_{d}=0.9, T=100 \mathrm{~ms} \mathrm{SNR}_{p}=-15 \mathrm{~dB}, \mathrm{SNR}_{s}=20$ $\mathrm{dB})$. From the figure, it can be noted that the achievable throughput with the proposed hybrid approach in both the cases (periodic sensing and simultaneous sensing/Tx) is higher than that of the conventional approach. It can be depicted that while increasing the interference threshold, the secondary throughput increases for both the proposed hybrid approaches.

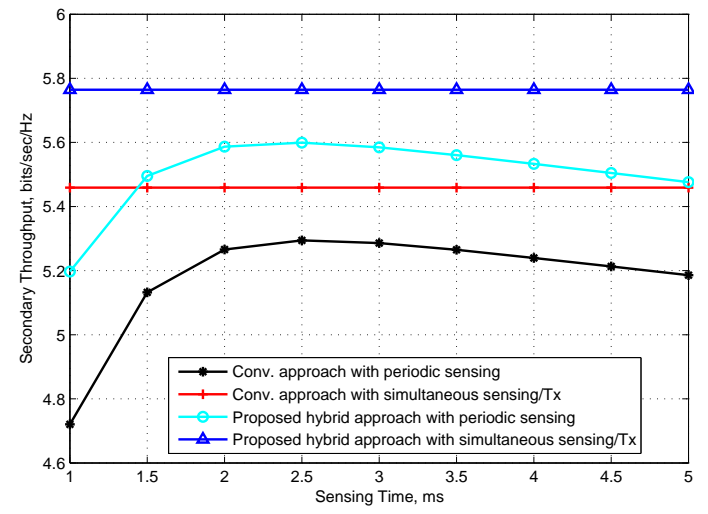

Fig. 5: Secondary throughput versus sensing time $\left(I_{T}=-130 \mathrm{dBW}\right.$, $\left.\overline{\mathcal{P}}_{d}=0.9, T=100 \mathrm{~ms}, \mathrm{SNR}_{p}=-15 \mathrm{~dB}, \mathrm{SNR}_{s}=20 \mathrm{~dB}\right)$

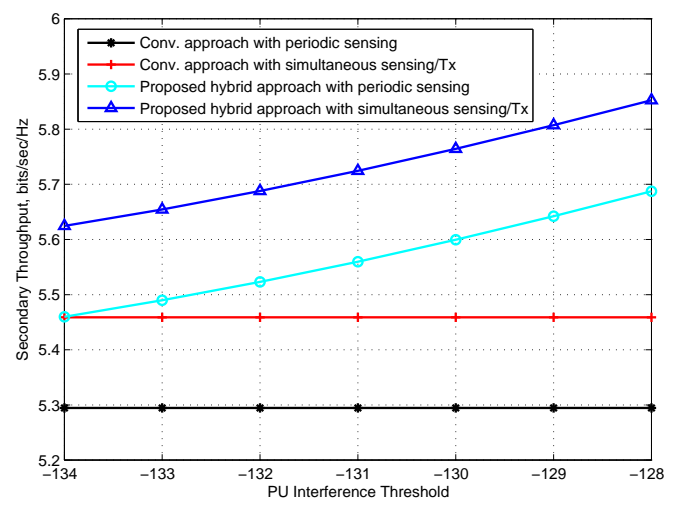

Fig. 6: Secondary throughput versus PU interference threshold for different approaches $\left(\overline{\mathcal{P}}_{d}=0.9, T=100 \mathrm{~ms}, \mathrm{SNR}_{p}=-15 \mathrm{~dB}\right.$, $\left.\mathrm{SNR}_{s}=20 \mathrm{~dB}, \tau=2.5 \mathrm{~ms}\right)$

It means that if the PU can tolerate more interference from the secondary system, secondary transmit power can be increased by guaranteeing the sufficient PU protection, hence resulting in the increased secondary throughput. Since conventional approaches do not consider achievable throughput from the underlay approach, no variation is observed on the secondary throughput with respect to the PU interference threshold.

Figure 7 depicts the secondary achievable throughput versus target probability of detection $\left(\overline{\mathcal{P}}_{d}\right)$ for all the considered approaches with parameters $\left(I_{T}=-130 \mathrm{dBW}, T=100 \mathrm{~ms}\right.$, $\mathrm{SNR}_{p}=-24 \mathrm{~dB}, \mathrm{SNR}_{s}=20 \mathrm{~dB}$ ). From the figure, it can be noted that the throughput decreases with the increase in the value of $\overline{\mathcal{P}}_{d}$ for the considered value of SNR. During simulation, it has been noted that the rate of throughput decrease with respect to $\overline{\mathcal{P}}_{d}$ increases as the received PU SNR decreases.

Figure 8 shows the secondary achievable throughput versus PU SNR for all the considered approaches with parameters $\left(I_{T}=-130 \mathrm{dBW}, T=100 \mathrm{~ms}, \mathrm{SNR}_{s}=20 \mathrm{~dB}, \tau=2.5 \mathrm{~ms}\right)$. From the figure, it can be noted that the secondary throughput increases with the increase in the received PU SNR for both the conventional and proposed hybrid approaches with the periodic sensing whereas for both the conventional and the proposed hybrid approaches with simultaneous sensing/Tx, the secondary throughput remains constant after some value of the received PU SNR. Furthermore, from the simulation 


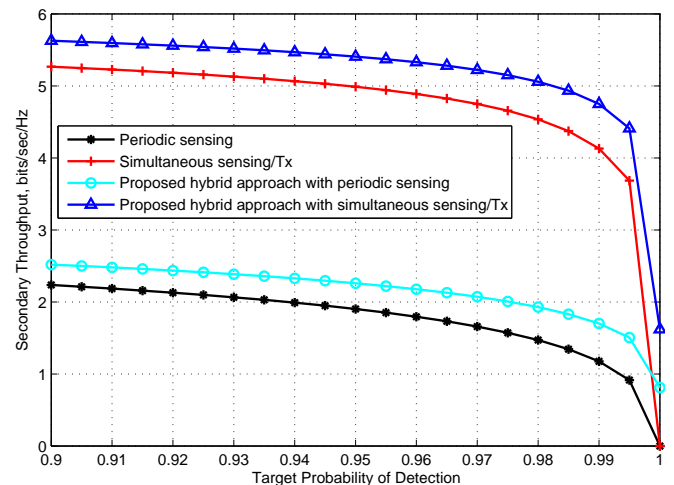

Fig. 7: Secondary throughput versus $\overline{\mathcal{P}}_{d}$ for different approaches $\left(I_{T}=-130 \mathrm{dBW}, T=100 \mathrm{~ms}, \mathrm{SNR}_{p}=-24 \mathrm{~dB}, \mathrm{SNR}_{s}=20 \mathrm{~dB}\right.$, $\tau=2.5 \mathrm{~ms})$

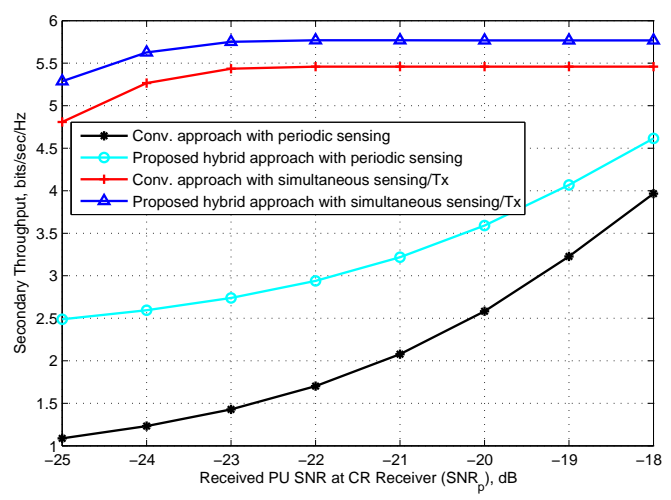

Fig. 8: Secondary throughput versus PU SNR for different approaches $\left(\overline{\mathcal{P}}_{d}=0.9, I_{T}=-130 \mathrm{dBW}, T=100 \mathrm{~ms}, \mathrm{SNR}_{s}=20 \mathrm{~dB}, \tau=2.5 \mathrm{~ms}\right)$ results, it can be noted that as the value of the received PU SNR increases, the performance gap between periodic sensing and simultaneous sensing/Tx decreases. Therefore, it can be concluded that at higher SNR regime, the periodic sensing scheme is advantageous from practical perspectives due to its lower complexity in comparison to the simultaneous sensing/Tx scheme.

\section{CONCLUSION}

A hybrid cognitive transceiver, which can overcome the drawbacks of SS only and underlay only approaches, has been proposed. The performance of the proposed approach has been evaluated in terms of the achievable throughput considering the periodic sensing and simultaneous sensing/transmission schemes. From the numerical results, it has been noted that the proposed hybrid approach for the periodic sensing case operates under a sensing-throughput tradeoff as it happens in the conventional periodic sensing approach. Furthermore, it can be concluded that the proposed hybrid scheme achieves significantly higher throughput than the conventional SS only approaches. We consider the extension of this architecture to the wideband context as our future work.

\section{ACKNOWLEDGEMENT}

This work was supported by the National Research Fund, Luxembourg under AFR (Aids Training-Research) grant for a $\mathrm{PhD}$ project (Reference 3069102 ) and the CORE projects "CO ${ }^{2}$ SAT" and "SEMIGOD".

\section{REFERENCES}

[1] A. Goldsmith, S. Jafar, I. Maric, and S. Srinivasa, "Breaking spectrum gridlock with cognitive radios: An information theoretic perspective," Proc. IEEE, vol. 97, no. 5, pp. 894 -914, May 2009.

[2] S. K. Sharma, S. Chatzinotas, and B. Ottersten, "Satellite cognitive communications: Interference modeling and techniques selection," in 6th ASMS/SPSC Conf., Sept. 2012, pp. 111-118.

[3] E. Axell, G. Leus, E. G. Larsson, and H. V. Poor, "Spectrum Sensing for Cognitive Radio : State-of-the-Art and Recent Advances," IEEE Signal Processing Magazine, vol. 29, no. 3, pp.101 -116, May 2012.

[4] S. K. Sharma, S. Chatzinotas, and B. Ottersten, "Exploiting polarization for spectrum sensing in cognitive SatComs," in 7th Int. Conf. CROWNCOM, June 2012, pp. $36-41$.

[5] M. Vu, N. Devroye, and V. Tarokh, "On the primary exclusive region of cognitive networks," IEEE Trans. Wireless Commun., vol. 8, no. 7, pp. $3380-3385$, July 2009.

[6] S. K. Sharma, S. Chatzinotas, and B. Ottersten, "Interference alignment for spectral coexistence of heterogeneous networks," EURASIP J. Wireless Commun. and Networking, vol. 46, 2013.

[7] S. K. Sharma, S. Chatzinotas, and B. Ottersten, "Transmit beamforming for spectral coexistence of satellite and terrestrial networks" in 8th Int. Conf. CROWNCOM, July 2013.

[8] Y.-C. Liang, Y. Zeng, E. Peh, and A. T. Hoang, "Sensing-throughput tradeoff for cognitive radio networks," IEEE Transactions on Wireless Communications, vol. 7, no. 4, pp. 1326-1337, 2008.

[9] S. Stotas, and A. Nallanathan, "On the throughput and spectrum sensing enhancement of opportunistic spectrum access cognitive radio networks," IEEE Trans. Wireless Commun., vol. 11, pp. 97-107, 2012.

[10] Z. Taheri, A. Taft, and S. Hoseini, "Cooperative spectrum sensing, power and throughput tradeoffs in cognitive radio systems," in Sixth Int. Symp. Telecommun., 2012, pp. 261-265.

[11] S. Senthuran, A. Anpalagan, and O. Das, "Throughput analysis of opportunistic access strategies in hybrid underlay and overlay cognitive radio networks," IEEE Trans. Wireless Commun., vol. 11, no. 6, pp. 2024-2035, 2012

[12] M. Cardenas-Juarez and M. Ghogho, "Spectrum sensing and throughput trade-off in cognitive radio under outage constraints over nakagami fading," IEEE Commun. Letters, vol. 15, no. 10, pp. 1110-1113, 2011.

[13] Y. Sharkasi, M. Ghogho, and D. McLernon, "Sensing-throughput tradeoff for OFDM-based cognitive radio under outage constraints," in Int. Symp. Wireless Commun. Systems, 2012, pp. 66-70.

[14] S. K. Sharma, S. Chatzinotas, and B. Ottersten, "SNR estimation for multi-dimensional cognitive receiver under correlated channel/noise," IEEE Trans. Wireless Commun., vol.12, no.12, pp. 6392-6405, 2013.

[15] X. Jiang, and et al, "On hybrid overlay-underlay dynamic spectrum access: Double-threshold energy detection and Markov model," IEEE Trans. Veh. Technol., vol. 62, no. 8, pp. 4078-4083, 2013.

[16] W. Cheng, X. Zhang, and H. Zhang, "Full duplex spectrum sensing in non-time-slotted cognitive radio networks," in Military Commun. Conf., 2011, pp. 1029-1034.

[17] M. Duarte, C. Dick, and A. Sabharwal, "Experiment-driven characterization of full-duplex wireless systems," IEEE Trans. Wireless Commun., vol. 11, no. 12, pp. 4296-4307, 2012.

[18] J. Vartiainen, and et al, "A blind signal localization and SNR estimation method," in IEEE Military Commun. Conf., 2006, pp. 1-7.

[19] S. K. Sharma, S. Chatzinotas, and B. Ottersten, "Eigenvalue based sensing and SNR estimation for cognitive radio in presence of noise correlation," IEEE Trans. Veh. Technol., vol. 42, no. 8, 2013.

[20] T. Tian, H. Iwai, and H. Sasaoka, "Pseudo BER based SNR estimation for energy detection scheme in cognitive radio," in IEEE 75th Veh. Technol. Conf., May 2012, pp. $1-5$.

[21] S. K. Sharma, S. Chatzinotas, and B. Ottersten, "Compressive SNR Estimation for Wideband Cognitive Radio under Correlated Scenarios", in IEEE WCNC, pp. 713-718, 2014.

[22] S. K. Sharma, S. Chatzinotas, and B. Ottersten, "Eigenvalue based SNR Estimation for Cognitive Radio in Presence of Channel Correlation", in IEEE Globecom pp. 1107-1112, 2013.

[23] S. M. Kay, "Fundamentals of Statistical Signal Processing," Volume 2, Detection Theory, First Edition, 1998.

[24] R. Couillet and M. Debbah, Random Matrix Methods for Wireless Communications, 1st ed. Cambridge University Press, 2011.

[25] S. J. Shellhammer, "Spectrum Sensing in IEEE 802.22," IAPR Workshop Cognitive Info. Processing, June 2008. 